\title{
Otomastoïdites aiguës dans le Service d'ORL du CHU Yalgadode Ouagadougou: à propos de 44 cas
}

\author{
R. W-L. OUEDRAOGO*, M. OUATTARA, C.L. BAMBARA, Y.M.C. GYEBRE, \\ B.P. OUEDRAOGO, M. SEREME, N. ZAGHRE, I. OUEDRAOGO et K. OUOBA
}

CHU-YO, Service d'ORL et de CCF, BP: 1220 Ouagadougou 11, Burkina Faso.

*Auteur correspondant ; E-mail : richarwendl@gmail.com

\section{RESUME}

Analyser les aspects diagnostiques et thérapeutiques des otomastoïdites dans le Service d'ORL du CHU-YO de Ouagadougou. Méthode : Il s'est agit d'une étude rétrospective de 10 ans, qui a porté sur 44 cas. Résultats: l'incidence annuelle était de 4,4 cas et l'âge moyen de 13,41 ans. Les mastoïdites étaient majoritairement fistulisées $(59,09 \%)$ et les principaux germes incriminés étaient Staphylococcus aureus (35,29\%), Proteus mirabilis $(23,53 \%)$ et Pseudomonas aeruginosa $(23,53 \%)$. Ils étaient surtout sensibles à la ciprofloxacine $(82,35 \%)$ et la ceftriaxone $(58,82 \%)$. Le traitement a été médico-chirurgical dans $86,36 \%$ des cas et l'évolution favorable dans $72,73 \%$. Douze cas $(27,27 \%)$ de décès par complication systémique et endocrânienne ont été enregistrés. Conclusion : Il convient de prioriser les mesures préventives et la prise en charge précoce des otites infectieuses.

(C) 2013 International Formulae Group. All rights reserved.

Mots clés: Otomastoïdite, épidémiologie, diagnostic, thérapeutique.

\section{INTRODUCTION}

Complication grave des otites moyennes mal ou non prises en charge, les otomastoïdites constituent une pathologie relativement fréquente. Elles touchent surtout les enfants d'âge scolaire avec des antécédents d'otites moyennes aigues ou chroniques. Elles sont diagnostiquées à un stade tardif dans notre contexte et posent surtout un problème de prise en charge adéquate. Si la révolution de l'antibiothérapie semble améliorer le pronostic de cette pathologie dans les pays développés (Trijolet, 2009), telle n'est pas le cas dans les pays en développement où les complications et les séquelles restent toujours d'actualité. Nous avons voulu à travers ce travail, rapporter notre expérience de prise en charge de 44 cas d'otomastoïdite aiguë dans le service d'ORL et de Chirurgie cervico-faciale du CHU-YO de Ouagadougou. Le but de cette étude était d'apprécier les aspects épidémiologiques, diagnostiques et thérapeutiques des otomastoïdites aiguës dans notre pratique courante.

\section{MATERIEL ET METHODES}

Cette étude rétrospective a porté sur 44 cas, colligés en 10 ans, de janvier 2002 à décembre 2011 dans le service d'ORL du CHU-YO de Ouagadougou. Ont été inclus 
dans l'étude, tous les patients reçus pour mastoïdite pendant la dite période dans le service et présentant un dossier clinique exploitable.

Les données ont été recueillies des dossiers cliniques des patients, des fiches de suivi et des registres de comptes rendus opératoires. Les variables d'étude ont été l'âge, le sexe, les antécédents, les manifestations cliniques, les données radiologiques et biologiques et les aspects thérapeutiques.

\section{RESULTATS}

\section{Données épidémiologiques}

Sur une période de 10 ans, 44 cas d'otomastoïdite ont été enregistrés dans le Service d'ORL du CHU-YO soit une incidence annuelle de 4,4 cas. Le sexe ratio était de 2,67 et l'âge moyen était de 13,41 ans +/- 11,91 avec des extrêmes de 01 et 41 ans. Les scolaires dans 16 cas $(36,36 \%)$ et les travailleurs champêtres dans 14 cas $(31,82 \%)$, ont constitué les couches sociales les plus touchées. Selon la résidence, 36 patients $(81,80 \%)$ provenaient des localités périphériques de Ouagadougou.

\section{Données diagnostiques}

Le délai moyen de consultation était de 16 jours avec des extrêmes de 03 et 36 jours. La tuméfaction retro-auriculaire dans 18 cas (40,91\%) et l'écoulement purulent dans 26 cas $(59,09 \%)$ étaient les principaux motifs de consultation. Les modes d'admission étaient dominés par la référence rencontrée dans 26 cas $(59,09 \%)$ et l'admission directe dans 14 cas $(31,82 \%)$. Les antécédents les plus fréquemment rencontrés ont été les otites moyennes aigües dans 24 cas $(54,55 \%)$, les otites moyennes chroniques dans 08 cas $(18,18 \%)$ et le diabète sucré dans 06 cas (13,64\%). Quatre cas (09,09\%) d'immunodépression à sérologie HIV positive et 02 cas $(4,55 \%)$ de corps étranger intratympanique ont été enregistrés.

La majorité des patients $(63,64 \%)$ présentait une altération de l'état général. Le diagnostic était clinique chez tous les patients et complété par la TDM du rocher dans 07 cas $(15,91 \%)$. La localisation des otomastoïdites était droite dans 16 cas $(36,36 \%)$, gauche dans 26 cas $(59,09 \%)$ et bilatérale dans 02 cas $(4,55 \%)$. Elles étaient non-extériorisées dans 06 cas $(13,64 \%)$, extériorisées non-fistulisées dans 12 cas $(27,27 \%)$ et fistulisées dans 26 cas $(59,09 \%)$. Une analyse bactériologique réalisée $(81,82 \%)$ a permis d'obtenir 06 cas $(16,67 \%)$ de bimicrobisme, 22 cas de monomicrobisme $(61,11 \%)$ et 08 cas de culture stérile $(22,22 \%)$. Les germes identifiés ont été répertoriés dans le Tableau 1.

L'antibiogramme réalisé dans les 34 cas a permis d'évaluer la sensibilité des germes aux antibiotiques couramment utilisés dans notre contexte (Figure 1).

\section{Données thérapeutiques et évolutifs}

Tous les patients ont bénéficié d'une prise en charge médicale qui a consisté en une antibiothérapie générale et intra-auriculaire associée à un antalgique. L'antibiothérapie a été systématiquement probabiliste dans tous les cas, puis secondairement adaptée à l'antibiogramme dans 34 cas $(77,27 \%)$. La bi antibiothérapie dans 32 cas $(72,73 \%)$ et la triple antibiothérapie dans 10 cas $(22,73 \%)$ ont été les protocoles thérapeutiques les plus utilisées dans notre série.

Le traitement chirurgical réalisé dans 38 cas $(86,36 \%)$ a consisté en une incision drainage de la collection dans 22 cas $(57,89 \%)$ et une mastoïdectomie dans 16 cas $(42,10 \%)$. Cette mastoïdectomie était partielle dans 06 cas et radicale dans 10 cas.

La cicatrisation a été obtenue dans 32 cas $(72,73 \%)$. Des séquelles ont été observées dans 24 cas soit $75 \%$ des guérisons. Ces séquelles étaient à type de surdité dans 12 cas (50\%) et de paralysie faciale périphérique dans 08 cas $(33,33 \%)$ et de sténose du conduit dans 04 cas $(16,67 \%)$. Douze cas de décès $(27,27 \%)$ suite à des complications à type de méningo-encéphalite dans 04 cas et de septicémie dans 08 cas ont été enregistrés. 
Tableau 1: Répartition des cas selon les germes identifiés.

\begin{tabular}{lcc}
\hline Germes & Fréquence & Pourcentage (\%) \\
\hline Staphylococcus aureus & 12 & 35,29 \\
Proteus mirabilis & 08 & 23,53 \\
Pseudomonas aeruginosa & 08 & 23,53 \\
Streptococuspneumoniae & 04 & 11,76 \\
Providencia & 02 & 5,88 \\
Total & $\mathbf{3 4}$ & $\mathbf{1 0 0}$ \\
\hline
\end{tabular}

Les différents germes identifiés ainsi que leur fréquence relative ont été répertoriés dans le tableau ci-dessus.

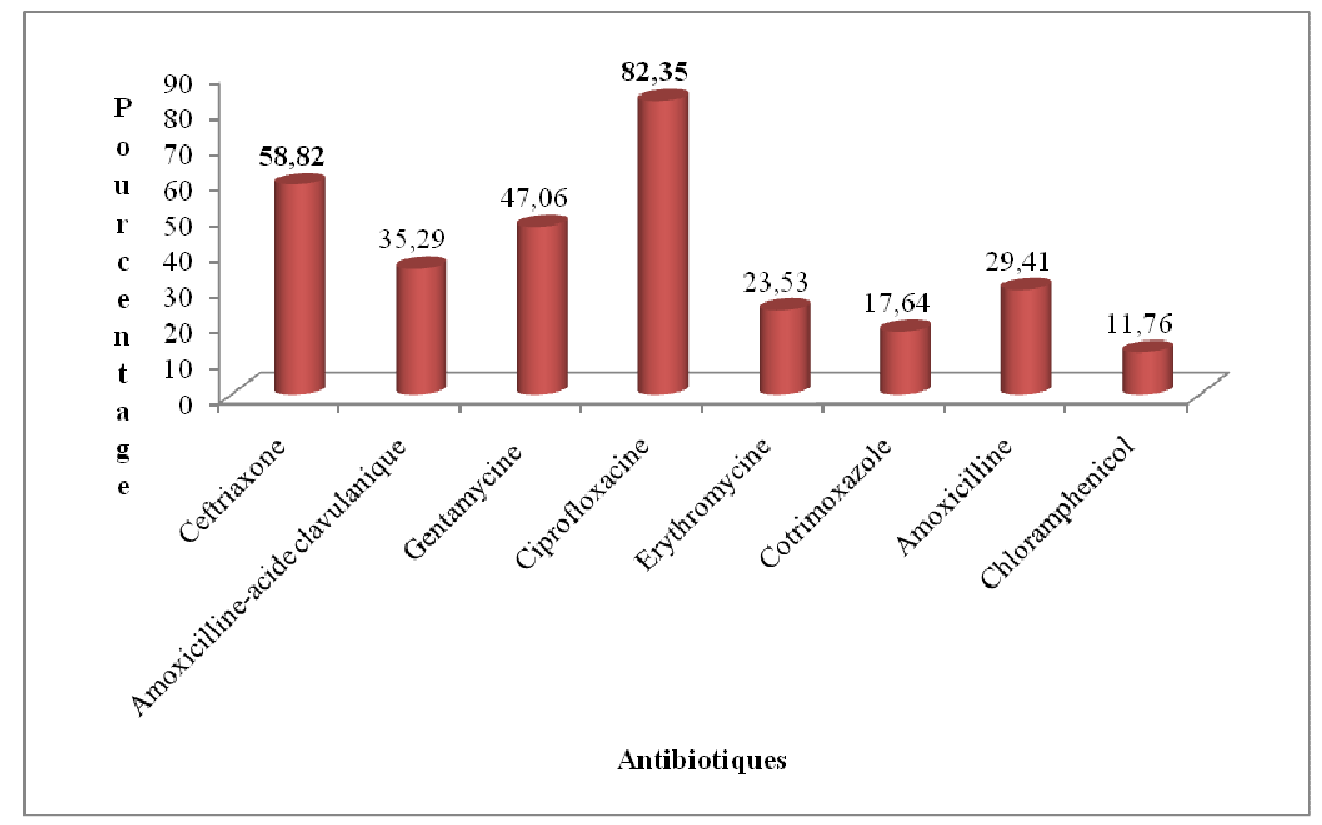

Figure 1: Répartition des antibiotiques couramment utilisés en fonction de leur degré de sensibilité. $(\mathrm{n}=34)$.

\section{DISCUSSION}

\section{Aspects épidémiologiques}

Les otomastoïdites constituent l'une des complications infectieuses post-otitiques les plus fréquentes en pratique ORL (Bordure, 2002; Morinière, 2003). Devenue de plus en plus rare dans les pays développés, 1,2 cas/an pour 100.0000 habitants en France et aux USA, cette pathologie demeure toujours d'actualité dans nos régions (Abada, 2009).
Ainsi une incidence annuelle de 4,4 cas a été enregistrée dans le service d'ORL du CHUYO de Ouagadougou. Le retard au diagnostic et à la prise en charge adéquate des otites bactériennes constitue un facteur déterminant dans la survenue de cette pathologie (Bordure, 2002; Heslop, 2006). Dans notre contexte, la faible accessibilité aux services de santé spécialisés, pourrait expliquer en partie l'importance de cette incidence. En effet, la 
majorité de nos patients $(81,80 \%)$ provenait des localités périphériques avec le plus souvent un traitement médical peu adapté apposé par un personnel médical ou paramédical omnipraticien. Dans notre série, les patients étaient surtout des enfants avec une moyenne d'âge de 13,41 ans et un sexe ratio de 2,67. Cette prédominance juvénile et masculine a été rapportée par la plupart des auteurs (Heslop, 2006 ; Abada, 2009 ; AbdelAziz, 2010). La fréquence élevée des infections ORL chez les enfants d'une part, et d'autre part la prédilection masculine des otites moyennes communément rapportée dans la littérature pourrait rendre compte de ces résultats.

\section{Aspects diagnostiques}

Dans notre contexte, à l'image des autres séries africaines (Abada, 2009), le diagnostic était tardif et par conséquent cliniquement évident. Ainsi, l'écoulement auriculaire $(59,09 \%)$ et la tuméfaction retroauriculaire $(40,91 \%)$ étaient les principaux motifs de consultation dans notre série. En effet, dans notre contexte de précarité économique, les patients ne consultent dans un centre de santé qu'après un long itinéraire thérapeutique extra-hospitalier laissant ainsi tout le temps à l'extériorisation voire à la fistulisation de la mastoïdite; ce qui expliquerait en parti le mauvais état général des patients $(63,64 \%)$ et la prédominance des otomastoïdites extériorisées $(27,27 \%)$ et fistulisées $(59,09 \%)$ dans notre série. Une analyse bactériologique des prélèvements auriculaires a permis de dresser le profil bactériologique dans 36 cas $(81,82 \%)$ de notre série. La prédominance du monomicrobisme rapportée dans les séries de Palma (2007) et Trijolet (2009) a été retrouvée dans notre série (22 cas). Staphylococcus aureus (35,29\%), Proteus mirabilis $(23,53 \%)$ et Pseudomonas aeruginosa $(23,53 \%)$ étaient les germes pathogènes les plus fréquemment rencontrés dans notre série. Ainsi le polymorphisme microbien semble être unanime dans la plupart des séries (Zanetti, 2006; Patricia, 2008; Abada, 2009). Ceci pourrait s'expliquer par la multiplicité des germes incriminés dans les otites moyennes, véritables pourvoyeuses d'otomastoïdites dans notre série à l'image des données de la littérature (Patricia, 2008 ; Abada, 2009). Ces otites moyennes étaient le plus souvent récidivantes, entretenues par un terrain immunodéficitaire ou un traitement inadéquat, responsable d'une sélection de germes résistants aux molécules courantes les plus accessibles. Ainsi l'érythromycine (23,53\%), le cotrimoxazole $(17,64 \%)$ et le chloramphénicol $(11,76 \%)$, molécules hors listes dans notre contexte et disponibles en contrefaçon «médicaments de la rue », se sont révélées peu actives contre les germes identifiés dans notre série. Par ailleurs, on note une bonne sensibilité de ces germes à la ciprofloxacine $(82,35 \%)$ et à la ceftriaxone $(58,82 \%)$. La précaution qu'entoure l'utilisation de ces molécules leur vaut une protection assurée. En effet, la ceftriaxone qui n'existe qu'en forme injectable donc en usage intra-hospitalier et la ciprofloxacine relativement contre-indiquée chez les enfants qui constituent le principal groupe sociodémographique concerné dans notre série, sont rarement utilisées en automédication préservant ainsi leur activité vis-à-vis de ces germes pathogènes.

\section{Aspects thérapeutiques et évolutifs}

Complication grave d'une pathologie banalisée à tort, les otomastoïdites constituent une urgence thérapeutique. Leur prise en charge est avant tout médicale et basée sur l'antibiothérapie primitivement ou secondairement adaptée à un antibiogramme (Bordure, 2002 ; Patricia, 2008; Abada, 
2009). Dans notre série tous les patients ont bénéficié d'une prise en charge médicale qui a consisté en une antibiothérapie générale et intra-auriculaire associée à un antalgique. L'antibiothérapie a été systématiquement probabiliste dans tous les cas, puis secondairement adaptée à l'antibiogramme (77,27\%). La bi-antibiothérapie $(72,73 \%)$ et la triple antibiothérapie $(22,73 \%)$ ont été les protocoles thérapeutiques, les plus utilisées dans notre série. Le but de ce traitement était de stériliser le foyer infectieux et surtout d'éviter la diffusion endocrânienne ou systémique, véritable hantise au cours de cette pathologie (Redaelli De Zinis, 2006). La stérilisation du foyer infectieux nécessite souvent une prise en charge chirurgicale complémentaire selon le stade évolutif de la maladie. Ainsi, un traitement chirurgical a été associé dans $86,36 \%$ des cas de notre série et a consisté en une incision drainage chirurgicale $(57,89 \%)$ et une mastoïdectomie $(42,10 \%)$ sous une bonne couverture antibiotique. Pour Trijolet (2009), la ponction drainage retro-auriculaire associée à la pose d'un aérateur transtympanique constitue une bonne alternative à la mastoïdectomie jugée souvent trop mutilante dans les otomastoïdites isolées sans diffusion endocrânienne. L'efficacité de la prise en charge est la seule garantie d'une bonne évolution de cette pathologie. Dans notre contexte, l'évolution a été satisfaisante avec l'obtention d'une guérison dans $72,73 \%$ des cas. Cependant des séquelles plus ou moins invalidantes ont été enregistrées dans 24 cas soit $75 \%$ des cas de guérison. La fréquence élevée des séquelles pourrait s'expliquer par le retard de la prise en charge. Aussi, la fragilité et la faible réversibilité des lésions des structures intrapétreuses notamment les nerfs (VII, VIII) et le labyrinthe sont le plus souvent à l'origine de séquelles fonctionnelles variables dominées par la surdité et la paralysie faciale périphérique. La létalité était élevée (27,27\%) et était surtout secondaire à des complications endocrâniennes et systémiques dans notre série.

\section{Conclusion}

Les otomastoïdites constituent une pathologie relativement fréquente dans notre contexte. Elles touchent surtout les enfants d'âge scolaire avec des antécédents d'otite moyenne, aigüe ou chronique. Elles restent hautement morbi-mortelles dans nos conditions d'exercice médical, caractérisées par le classique retard à la prise en charge et l'insuffisance du plateau technique. Si l'évolution de l'antibiothérapie adaptée a permis d'améliorer le pronostic vital de cette pathologie, le pronostic fonctionnel séquellaire demeure une préoccupation majeure dans notre contexte. Il convient donc de prioriser les mesures préventives et la prise en charge précoce des otites infectieuses, qu'elles soient aiguës ou chroniques.

\section{REFERENCES}

Abada RL, Mansouri I, Maamri M, Kadiri F. 2009. Complications des otites moyennes chroniques. Annales d'Otolaryngologie et de Chirurgie Cervico-Faciale, 126: 1-5.

Abdel-Aziz M, EL-Hoshy H. 2010. Acute mastoiditis: A one year study in the pediatric hospital of Cairo University. BMC Ear. Nose Throat. Disord., 4: 1011.

Bordure P, Malard O. 2002. Chirurgie des cavités postérieures de l'oreille moyenne et antro-atticotomies. In Encycl. Méd. Chir. Editions Scientifiques et Médicales Elsevier SAS : Paris ; $12 \mathrm{p}$.

Heslop A, Ovesen T. 2006. Severe acute middle ear infections: microbiology and treatment. Int. J. Pediatr. Otorhinolaryngol., 70(10): 1811-1816. 
Morinière S, Lanotte P, Celebi Z, Ployet MJ, Robier A, Lescanne A. 2003. Mastoïdite aiguë de l'enfant. Presse Méd., 32: 14451449.

Palma S, Fiumana E, Borgonzoni M, Bovo R, Rosignoli M, Martini A. 2007. Acute mastoiditis in children: the "Ferrara" experience. Int. J. Pediatr. Otorhinolaryngol., 71(11): 1663-1669.

Patricia De Pma, Janini OM, Felipe BM, Anderson De Sa, Andréia MA, Shiro T. 2008. Squamozygomatic mastoiditis. Rev Bras Otorrinolaringol., 74(4): 617-620.

Redaelli de Zinis LO, Gasparotti R, Campovecchi C, Annibale G, Barezzani
MG. 2006. Jugular vein thrombosis associated with acute mastoiditis in a pediatric age. Otol. Neurotol. Internal, 27(7): 937-944.

TrijoletJ-P, Bakhos D, Lanotte P, Pondaven S, Lescanne E. 2009. Mastoïdite aiguë extériorisée chez l'enfant: la mastoïdectomie peut-elle être évitée? Annales d'Otolaryngologie et de Chirurgie Cervico-Faciale, 126(4): 169174.

Zanetti D, Nassif N. 2006. Indications for surgery in acute mastoiditis and their complications in children. Int. J. Pediatr. Otorhinolaryngol., 70(7): 1175-1182. 